\title{
ON THE DETERMINANT OF AN INTEGRAL LATTICE GENERATED BY RATIONAL APPROXIMANTS OF THE EULER CONSTANT
}

\author{
A. I. APTEKAREV AND D. N. TULYAKOV
}

\begin{abstract}
We investigate rational approximants of the Euler constant constructed using a certain system of jointly orthogonal polynomials and "averaging" such approximants of mediant type. The properties of such approximants are related to the properties of an integral lattice in $\mathbb{R}^{3}$ constructed from recurrently generated sequences. We also obtain estimates on the metric properties of a reduced basis, which imply that the $\gamma$-forms with coefficients constructed from basis vectors of the lattice tend to zero. The question of whether the Euler constant is irrational is reduced to a property of the bases of lattices.
\end{abstract}

\section{INTRODUCTION}

The papers in [1] dealt with some aspects of rational approximants $p_{n} / q_{n} \rightarrow \gamma, n \rightarrow \infty$, of the Euler constant

$$
\gamma:=\lim _{n \rightarrow \infty}\left(\sum_{k=1}^{n} \frac{1}{k}-\ln n\right) .
$$

Those constructs are based on a certain type of functional Hermite-Padé approximants from [2. Their numerators and denominators satisfy the following recurrence relation (for the proof, see [3]-[5]):

$$
\begin{aligned}
(16 n-15) q_{n+1}= & \left(128 n^{3}+40 n^{2}-82 n-45\right) q_{n} \\
& -n^{2}\left(256 n^{3}-240 n^{2}+64 n-7\right) q_{n-1} \\
& +n^{2}(n-1)^{2}(16 n+1) q_{n-2},
\end{aligned}
$$

with initial conditions

$$
\begin{aligned}
& p_{0}=0, \quad p_{1}=2, \quad p_{2}=31, \\
& q_{0}=1, \quad q_{1}=3, \quad q_{2}=50 .
\end{aligned}
$$

Moreover, we also have [6] the following asymptotic formulas:

$$
\begin{gathered}
q_{n}=(2 n) ! \frac{e^{\sqrt{2 n}}}{\sqrt[4]{n}}\left(\frac{1}{\sqrt{\pi}(4 e)^{3 / 8}}+O\left(n^{-1 / 2}\right)\right), \\
f_{n}:=p_{n}-\gamma q_{n}=(2 n) ! \frac{e^{-\sqrt{2 n}}}{\sqrt[4]{n}}\left(\frac{2 \sqrt{\pi}}{(4 e)^{3 / 8}}+O\left(n^{-1 / 2}\right)\right) .
\end{gathered}
$$

Recently [7, for those rational approximants, a system of recurrence relations with leading coefficient one was found (which shows that $p_{n}$ and $q_{n}$ defined by (1.1)-(1.2) are

2000 Mathematics Subject Classification. Primary 11J72; Secondary 33C45, 41A21.

Partially supported by RFFI, Project No. 08-01-00179, Program No. 1 OMN RAN, and the Support Program for Leading Scientific Schools (Project NSh-3906.2008.1). 
integers). Also recently, Rivoal [8] found another example of rational approximants of the Euler constant given by recurrence relations.

In the present paper we consider three-dimensional lattices $L$ of integral vectors $\{\vec{x}\}$ of solutions of the Diophantine system of congruences

$$
\vec{x} \in L:\left\{\begin{aligned}
\left\langle\vec{x}, \vec{p}^{(n)}\right\rangle & \equiv 0 \bmod D_{n}, \\
\left\langle\vec{x}, \vec{q}^{(n)}\right\rangle & \equiv 0 \bmod D_{n},
\end{aligned}\right.
$$

where the coordinates of the vectors

$$
\vec{p}^{(n)}:=\left(p_{n}, p_{n-1}, p_{n-2}\right), \quad \vec{q}^{(n)}:=\left(q_{n}, q_{n-1}, q_{n-2}\right)
$$

are the numerators and denominators of the rational approximants of $\gamma$ generated by (1.1) - (1.2). Also, $D_{n}$ in (1.4) is a certain fixed sequence of natural numbers, $n \in \mathbb{N}$, and $\langle\cdot, \cdot\rangle$ is the scalar product in $R^{3}$.

The motivation for this problem should be clear. We are interested in the reducible (by $D_{n}$ ) "mediants" of three consecutive rational approximants of $\gamma$ :

$$
\frac{x_{0} p_{n}+x_{1} p_{n-1}+x_{2} p_{n-2}}{x_{0} q_{n}+x_{1} q_{n-1}+x_{2} q_{n-2}}=\frac{D_{n} P_{n}}{D_{n} Q_{n}},
$$

such that we can get rid of the factorial growth in the $\gamma$-forms $f_{n}$ (see (1.3)) by switching to the $\gamma$-forms $F_{n}$,

where $\vec{f}^{(n)}=\vec{p}^{(n)}-\gamma \vec{q}^{(n)}$ (see (1.5)).

$$
F_{n}:=P_{n}-\gamma Q_{n}=\frac{1}{D_{n}}\left\langle\vec{x}, \vec{f}^{(n)}\right\rangle,
$$

The main result of this paper is an expression for the determinant of $L$. Let

$$
B=\left\{\vec{b}^{(0)}, \vec{b}^{(1)}, \vec{b}^{(2)}\right\}, \quad \vec{b}^{(k)}=\left(b_{0}^{(k)}, b_{1}^{(k)}, b_{2}^{(k)}\right), \quad k=0,1,2,
$$

be a basis of $L$ :

$$
L=\operatorname{span}\{B\} .
$$

By definition, the determinant of $L$ is

$$
\operatorname{det}(L):= \pm\left|\begin{array}{ccc}
b_{0}^{(0)} & b_{1}^{(0)} & b_{2}^{(0)} \\
b_{0}^{(1)} & b_{1}^{(1)} & b_{2}^{(1)} \\
b_{0}^{(2)} & b_{1}^{(2)} & b_{2}^{(2)}
\end{array}\right|, \quad \operatorname{det}(L)>0 .
$$

It does not depend on the basis. Let

$$
\begin{aligned}
d_{n} & :=\operatorname{GCD}\left\{\operatorname{det}\left(\begin{array}{cc}
p_{n-1}, & p_{n-2} \\
q_{n-1}, & q_{n-2}
\end{array}\right), \operatorname{det}\left(\begin{array}{cc}
p_{n}, & p_{n-2} \\
q_{n}, & q_{n-2}
\end{array}\right), \operatorname{det}\left(\begin{array}{ll}
p_{n}, & p_{n-1} \\
q_{n}, & q_{n-1}
\end{array}\right)\right\}, \\
\Delta_{n} & :=[(n-1) !]^{2}[(n-2) !]^{2}(16 n-15), \quad d_{n}^{0}:=[(n-2) !]^{2} .
\end{aligned}
$$

We then have

Theorem 1. A) Suppose the parameter $D_{n}$ of (1.4) is divisible by $d_{n}$ (see (1.7)):

$$
D_{n} \equiv 0 \quad \bmod d_{n} .
$$

Then

$$
\operatorname{det}(L)=\frac{D_{n}^{2}}{d_{n}} .
$$

B) For lattices (1.4) determined by the vectors (1.5) generated by recurrence relations (1.1) -(1.2) we have

$$
\Delta_{n} \equiv 0 \quad \bmod d_{n}, \quad d_{n} \equiv 0 \quad \bmod d_{n}^{0} .
$$


Thus, to use formula (1.8) for the determinant of the lattice (1.4)-(1.5), the parameter $D_{n}$ should be of the form

$$
D_{n}=k_{n} \Delta_{n}, \quad k_{n} \in \mathbb{N} .
$$

In that case, the determinant of the lattice would have an upper bound

$$
\operatorname{det}(L) \leq \frac{D_{n}^{2}}{d_{n}^{0}}
$$

Notice that (1.11) is probably sharp since computational experiments yield the following expression for $d_{n}$ :

$$
d_{n}=c d_{n}^{0}, \quad c=\left\{\begin{array}{lll}
3, & n \equiv 1 & \bmod 3 \\
1, & n \neq 1 & \bmod 3 .
\end{array}\right.
$$

\section{Minimal vectors in $L$ and irrationality of $\gamma$}

We now return to the original motivation: construction of nonzero $\gamma$-forms (1.6) converging to zero. It is clear that

$$
\exists \vec{x} \in L:\|\vec{x}\| \leq \operatorname{Const}(\operatorname{det}(L))^{1 / 3}=\text { Const } \frac{D_{n}^{2 / 3}}{d_{n}^{1 / 3}},
$$

where the absolute constant Const does not depend on $n$. For the corresponding $\gamma$ forms $F_{n}$ (see (1.6)) we have rough estimates (taking account of (1.8)):

$$
\left|F_{n}\right|:=\left|P_{n}-\gamma Q_{n}\right| \leq \frac{\|\vec{x}\|\left\|\vec{f}^{(n)}\right\|}{D_{n}} \leq \text { Const } \frac{\left\|\vec{f}^{(n)}\right\|}{\left(D_{n} d_{n}\right)^{1 / 3}} .
$$

Since (see (1.3))

$$
\left\|\vec{f}^{(n)}\right\| \sim \frac{2 \sqrt{\pi}}{(4 e)^{3 / 8}}(2 n) ! \frac{e^{-\sqrt{2 n}}}{\sqrt[4]{n}},
$$

we can, in view of (1.9), choose $D_{n}$ such that

$$
\left\{\begin{array}{l}
D_{n} \equiv 0 \quad \bmod \Delta_{n}, \\
D_{n} \geq D_{n}^{(0)}:=\frac{[(2 n) !]^{3}}{[(n-2) !]^{2}} .
\end{array}\right.
$$

This can be done, since $D_{n}=k_{n} \Delta_{n}$ (1.10), by assuming

$$
k_{n} \geq\left[\left(\frac{\text { Const } 2 \sqrt{\pi}}{(4 e)^{3 / 8} \sqrt[4]{n}}\right)^{3} \cdot \frac{D_{n}^{(0)}}{\Delta_{n}}\right],
$$

(where [.] is the integer part). Indeed (see (1.7)), by Stirling's formula,

$$
\left[\frac{D_{n}^{(0)}}{\Delta_{n}}\right] \sim\left[\frac{4^{3 n}}{16 \pi^{3 / 2} n^{12.5}}\right]=: k_{n}^{0} .
$$

Hence, when

$$
k_{n} \geq\left[\frac{\text { Const }^{3}}{2(4 e)^{3 / 8}} \cdot \frac{4^{3 n}}{n^{13.25}}\right],
$$

we have that the vectors $\vec{x}$ satisfying (2.1) generate $\gamma$-forms converging to zero, with integer coefficients $F_{n}$ :

$$
\left|F_{n}\right| \leq \widetilde{\text { Const }} \cdot \frac{e^{-\sqrt{2 n}}}{\sqrt[4]{n}}
$$


However, to prove that such $\gamma$-forms are not zero (i.e., the vectors $\vec{x}$ and $\vec{f}^{(n)}$ are not orthogonal) is arguably a problem as difficult as the original one, despite the ostensible "simplifications" similar to this one: since all the coordinates of $\vec{f}^{(n)}$ are positive (see (1.3)), it suffices to find a vector $\vec{x}$ in $L$ satisfying (2.1) and having coordinates of the same sign.

One can take another approach. For example, we can find two linearly independent $\gamma$-forms $F_{n}^{(1)}$ and $F_{n}^{(2)}$ (see (1.3) ) (with nonzero $P_{n}$ and $Q_{n}$ ) converging to zero when $n \rightarrow$ $\infty$. If one of those forms becomes zero, then $\gamma$ is rational, and therefore, the other form must also vanish beginning with some $n$, which contradicts their linear independence. To see how such two forms may be constructed, consider the structure of $L(1.4)$ in more detail.

The lattice $L$ contains the one-dimensional sublattice of the "annihilating" vectors

$$
\left\{k \cdot \vec{V}_{0}\right\}_{k \in \mathbb{Z}}:\left\{\begin{array}{l}
\left\langle\vec{V}_{0}, \vec{p}^{(n)}\right\rangle=0, \\
\left\langle\vec{V}_{0}, \vec{q}^{(n)}\right\rangle=0,
\end{array}\right.
$$

where the "annihilating" vector $\overrightarrow{V_{0}}$ equals

$$
\vec{V}_{0}=\frac{\left[\vec{p}^{(n)} \times \vec{q}^{(n)}\right]}{d_{n}}=\frac{\left(\operatorname{det}\left(\begin{array}{cc}
p_{n-1}, & p_{n-2} \\
q_{n-1}, & q_{n-2}
\end{array}\right),-\operatorname{det}\left(\begin{array}{c}
p_{n}, p_{n-2} \\
q_{n}, q_{n-2}
\end{array}\right), \operatorname{det}\left(\begin{array}{c}
p_{n}, p_{n-1} \\
q_{n}, q_{n-1}
\end{array}\right)\right)}{d_{n}} .
$$

Moreover, the asymptotics of its norm is given by

\section{Proposition 1.}

$$
\left\|\overrightarrow{V_{0}}\right\|=\frac{\sqrt{2}}{(4 e)^{3 / 4}} \frac{[(2 n) !]^{2}}{n^{3} d_{n}}\left(1+O\left(\frac{1}{\sqrt{n}}\right)\right) .
$$

The lattice $L$ can be thought of as follows. In the plane $\mathcal{P}:=\operatorname{span}\left\{\vec{p}^{(n)}, \vec{q}^{(n)}\right\}$ consider the two-dimensional lattice $L_{\mathcal{P}}$ which is the projection of $L$ to $\mathcal{P}$. Through each point of $L_{\mathcal{P}}$ there passes a line perpendicular to $\mathcal{P}$, and the points of $L$ are located along those lines with step $\left\|\overrightarrow{V_{0}}\right\|$. More precisely, the lines determined by the vectors $\vec{p}^{(n)}$ and $\vec{q}^{(n)}$ are broken into intervals of lengths $D_{n} /\left\|\vec{p}^{(n)}\right\|$ and $D_{n} /\left\|\vec{q}^{(n)}\right\|$, respectively, and the lines perpendicular to $\vec{p}^{(n)}$ and $\vec{q}^{(n)}$ and passing through the endpoints of those intervals form the lattice $L_{\mathcal{P}}$ which is the projection of $L$.

It is now clear that, choosing the $D_{n}$ in (2.3) that grow too fast, we have that the vectors in the lattice satisfying (2.1) will be proportional to the "annihilating" vector $\vec{V}_{0}$. We remark that when $D_{n} \sim D_{n}^{(0)}$, it follows from (2.7), (1.8), and (2.3), in view of (1.12), that

$$
\left\|\overrightarrow{V_{0}}\right\| \asymp \frac{1}{n^{3}}(\operatorname{det} L)^{1 / 3} .
$$

Thus the choice of the lattice parameter $D_{n}$ is determined by two processes: $D_{n}$ should be "large" enough to have (1.6) decrease, and on the other hand, $D_{n}$ should be "small" enough to guarantee, in (1.6), the existence of "nonannihilating" vectors $\vec{x}$ with small norm $\left(\right.$ of order $\left.(\operatorname{det} L)^{1 / 3}\right)$.

To estimate vectors with "small" norm it is convenient to work with a minimal, or reduced, basis as defined in [9]. Without going into details (see [9]), we just mention the main estimate for the lengths of the vectors in such a basis. For any (three-dimensional in our case) integral lattice there exists a basis $b=\left\{\vec{b}^{(0)}, \vec{b}^{(1)}, \vec{b}^{(2)}\right\}$ such that

$$
\left\|\vec{b}^{(0)}\right\| \leq 2\left\|\vec{x}^{(0)}\right\|, \quad\left\|\vec{b}^{(j)}\right\| \leq 2 \max \left\{\left\|\vec{x}^{(0)}\right\|, \ldots,\left\|\vec{x}^{(i)}\right\|\right\}, \quad i=1,2, \quad j \leq i,
$$


where $\left(\vec{x}^{(0)}, \ldots, \vec{x}^{(i)}\right)$ are any linearly independent vectors of $L$. One can say that $\vec{b}^{(0)}$ is a "minimal" vector in the lattice and that the basis consists of linearly independent vectors with "minimal" lengths such that

$$
\begin{gathered}
\left\|\vec{b}^{(0)}\right\| \leq \sqrt{2} \operatorname{det}(L)^{1 / 3}, \quad\left\|\vec{b}^{(1)}\right\| \leq 2^{3 / 4} \frac{\operatorname{det}(L)^{1 / 2}}{\left\|\vec{b}^{(0)}\right\|^{1 / 2}}, \\
\left\|\vec{b}^{(2)}\right\| \leq 2^{3 / 2} \frac{\operatorname{det}(L)}{\left\|\vec{b}^{(0)}\right\|\left\|\vec{b}^{(1)}\right\|} .
\end{gathered}
$$

The constants in the right-hand sides of the inequalities estimating the lengths of the vectors in a reduced basis depend only on the dimension of the lattice. Any basis $B$ of $L$ can be made into a reduced basis $b$ using the $L L L$ algorithm (see [7]) with a polynomial (in the dimension of the lattice) and logarithmic (in the lengths of the vectors in the original basis $B$ ) complexity.

The estimates in (2.9) are fairly good from above, but to use them we also need good estimates from below, which we do not yet have. Without such estimates from below we can only state a conditional result.

Theorem 2 (conditional). Let $L$ be the lattice (1.4)-(1.5) generated by rational approximations (1.1)-(1.2), and suppose that the lattice parameter $D_{n}$ is given by (see (2.4)):

$$
D_{n}=k_{n}^{(0)} \Delta_{n} \sim D_{n}^{(0)}:=\frac{[(2 n) !]^{3}}{(n !)^{2}}
$$

If the "annihilating" vector $V_{0}$ of the lattice is minimal:

$$
V_{0}= \pm \vec{b}^{(0)},
$$

then the vectors $\vec{b}^{(1)}, \vec{b}^{(2)}$ generate integral $\gamma$-forms (1.6)

$$
F_{n}^{(j)}:=P_{n}^{(j)}-\gamma Q_{n}^{(j)}:=\frac{1}{D_{n}}\left\langle\vec{b}^{(j)}, \vec{f}^{(n)}\right\rangle, \quad j=1,2,
$$

such that

$$
\left|\begin{array}{ll}
P_{n}^{(1)} & Q_{n}^{(1)} \\
P_{n}^{(2)} & Q_{n}^{(2)}
\end{array}\right|=1
$$

and for some (explicitly determined) $c>0$ we have

$$
\left|F_{n}^{(j)}\right| \leq O\left(n^{c} e^{-\sqrt{2 n}}\right) \quad n \rightarrow \infty ; \quad j=1,2 .
$$

We remark that our computations of a reduced basis $b$ with $n \leq 5000$ show that in this case (2.10) $\Rightarrow(2.11)$. Using rough estimates from below we have the following theorem.

Theorem 3. Choosing the parameter of $L$ (1.4)-(1.5) as in (1.1)-(1.2),

$$
D_{n}=D_{n}^{(1)}:=\left[e^{\sqrt{2 n}} n^{3 / 4}\right] k_{n}^{(0)} \Delta_{n} \quad([\cdot] \text { denotes the integer part })
$$

guarantees (2.11), but the forms (2.12) (still satisfying (2.13)) can be estimated from above as follows:

$$
\begin{array}{ll}
\exists c>0 & \left|F^{(1)}\right| \leq O\left(n^{c} e^{-\sqrt{2 n}}\right) \\
& \left|F^{(2)}\right| \leq O\left(n^{c}\right)
\end{array}
$$

We remark that computations show that the second $\gamma$-form in (2.14) is indeed growing slowly when $n \leq 5000$.

Thus, the conjecture on irrationality of the Euler constant $\gamma$ can be reformulated as a conjecture on the implication (2.10) $\Rightarrow$ (2.11). 
TABLE 1

\begin{tabular}{|c|c|c|c|c|}
\hline$n$ & $\frac{P_{n}^{(1)}}{Q_{n}^{(1)}}$ & $F_{n}^{(1)}$ & $\frac{P_{n}^{(2)}}{Q_{n}^{(2)}}$ & $F_{n}^{(2)}$ \\
\hline \hline 10 & $\frac{327}{566}$ & -0.29593 & $\frac{955}{1653}$ & -0.86250 \\
\hline 50 & $\frac{505548}{875839}$ & 0.00926 & $\frac{1357157}{2351213}$ & 0.02487 \\
\hline 100 & $\frac{55335264}{95865839}$ & -0.00027 & $\frac{201557807}{349189773}$ & -0.00098 \\
\hline 200 & $\frac{74764878578}{129526766379}$ & $0.3 \cdot 10^{-5}$ & $\frac{147419421621}{255397472011}$ & $0.6 \cdot 10^{-5}$ \\
\hline
\end{tabular}

We now list the computed rational approximants of the Euler constant given by (2.12), (2.10) (see Table1).

\section{Proof of Theorem 1}

Proof of A). Set

$$
A:=\left(\begin{array}{ccccc}
p_{n} & q_{n} & 1 & 0 & 0 \\
p_{n-1} & q_{n-1} & 0 & 1 & 0 \\
p_{n-2} & q_{n-2} & 0 & 0 & 1 \\
D_{n} & 0 & 0 & 0 & 0 \\
0 & D_{n} & 0 & 0 & 0
\end{array}\right) .
$$

The system of congruences (1.4)-(1.5) can be rewritten as

$$
\left(x_{1}, x_{2}, x_{3},-P,-Q\right) \cdot A=\left(0,0, x_{1}, x_{2}, x_{3}\right) ;
$$

i.e., for any fixed integers $P$ and $Q$ we need to find integer solutions of this equation.

Let $u$ and $v$ be two arbitrary integers and let

$$
a, b, r \in \mathbb{Z}: \quad r:=\operatorname{GCD}(u, v)=a u+b v .
$$

Consider an elementary transformation

$$
T:=\left(\begin{array}{ccccc}
1 & 0 & 0 & 0 & 0 \\
0 & 1 & 0 & 0 & 0 \\
0 & 0 & a & b & 0 \\
0 & 0 & -\frac{v}{r} & \frac{u}{r} & 0 \\
0 & 0 & 0 & 0 & 1
\end{array}\right)
$$

that affects two rows (in our example these are rows 3 and 4). Such transformations have determinant 1 and preserve the integrality of the matrices. They only change the two affected rows as follows:

$$
T \cdot\left(\begin{array}{ccccc}
\nabla & \nabla & \nabla & \nabla & \nabla \\
\triangle & \triangle & \triangle & \triangle & \triangle \\
0 & u & * & * & * \\
0 & v & \square & \square & \square \\
\diamond & \diamond & \diamond & \diamond & \diamond
\end{array}\right)=\left(\begin{array}{ccccc}
\nabla & \nabla & \nabla & \nabla & \nabla \\
\triangle & \triangle & \triangle & \triangle & \triangle \\
0 & (a u+b v) & a *+b \square & \bullet & \bullet \\
0 & \left(\frac{-v u+u v}{r}\right) & \left(\frac{-v *+u \square}{r}\right) & \bullet & \bullet \\
\diamond & \diamond & \diamond & \diamond
\end{array}\right) .
$$


Now, using such transformations, we transform $A$ to a row-echelon form

$$
T_{N} T_{N-1} \ldots T_{1} A=R \text {. }
$$

Since $A$ is nondegenerate ( $\operatorname{det} A=D_{n}^{2} \neq 0$ ), $R$ is upper-triangular:

$$
R=\left(\begin{array}{ccccc}
r_{11} & r_{12} & r_{13} & r_{14} & r_{15} \\
& r_{22} & r_{23} & r_{24} & r_{25} \\
& & r_{33} & r_{34} & r_{35} \\
& 0 & & r_{44} & r_{45} \\
& & & & r_{55}
\end{array}\right) .
$$

The transformation from $A$ to $R$ makes (3.2) into

$$
\begin{array}{r}
\left(x_{1}, x_{2}, x_{3},-P,-Q\right) T_{1}^{-1} \circ T_{2}^{-1} \circ \cdots \circ T_{N}^{-1} \circ R \\
\quad=\left(\alpha_{1}, \alpha_{2}, \alpha_{3}, \alpha_{4}, \alpha_{5}\right) R=\left(0,0, x_{1}, x_{2}, x_{3}\right),
\end{array}
$$

where $\alpha_{j} \in \mathbb{Z}, j=1, \ldots, 5$, and, since $R$ is triangular, $\alpha_{1}=\alpha_{2}=0$. Thus for any vector $\vec{x}=\left(x_{1}, x_{2}, x_{3}\right)$ in $L$ we have

$$
\vec{x}=\left(\alpha_{3}, \alpha_{4}, \alpha_{5}\right)\left(\begin{array}{ccc}
r_{33} & r_{34} & r_{35} \\
0 & r_{44} & r_{45} \\
0 & 0 & r_{55}
\end{array}\right)=\alpha_{3}\left(r_{33}, r_{34}, r_{35}\right)+\alpha_{4}\left(0, r_{44}, r_{45}\right)+\alpha_{5}\left(0,0, r_{55}\right) .
$$

Similarly, we have the converse. If the row $\left(0,0, x_{1}, x_{2}, x_{3}\right)$ is an integer linear combination of rows of $R$, then it is an integer linear combination of rows of $A$. The corresponding coefficients are $\left(x_{1}, x_{2}, x_{3},-P,-Q\right)$, where $P$ and $Q$ are some integers.

Thus, the vectors

$$
\left\{\begin{array}{l}
\vec{B}^{(0)}:=\left(r_{33}, r_{34}, r_{35}\right), \\
\vec{B}^{(1)}:=\left(0, r_{44}, r_{45}\right), \\
\vec{B}^{(2)}:=\left(0,0, r_{55}\right)
\end{array}\right.
$$

form a (triangular) basis of $L$ and

$$
\operatorname{det}(L)=r_{33} r_{44} r_{55}
$$

Notice that (since the determinant of $T$ is 1 ) $\operatorname{det} R=\operatorname{det} A=D_{n}^{2}$, and therefore

$$
\operatorname{det}(L)=\frac{D_{n}^{2}}{r_{11} r_{22}}
$$

It remains to find $r_{11} \cdot r_{22}$. We give a general argument. Let

$$
A=\left(a_{i j}\right)_{i, j=1}^{m}
$$

be the original matrix (3.1) (in our case $m=5$ ). Pick an arbitrary $k \in\{1, \ldots, m\}$ and consider all possible minors of order $k$ which can be formed from the elements of the first $k$ columns, i.e., the quantities $\operatorname{det}\left(a_{i j}\right), i \in u, j \in\{1, \ldots, k\}$ indexed by subsets $u \subset\{1, \ldots, m\}$ with $k$ elements. Under each elementary transformation $T_{j}$ any such minor either remains the same or is an integer linear combination of some minors from the previous transformation. Hence the principal minor $\prod_{i=1}^{k} r_{i i}$ of $R$ is a linear combination of minors of $A$. On the other hand, all minors of $A$ are either zero or $\prod_{i=1}^{k} r_{i i}$. Since all transformations applied to $A$ are invertible (and their inverses also have integer entries), any minor $\operatorname{det}\left(a_{i j}\right), i \in u, j \in\{1, \ldots, k\}$ is an integer linear combination of minors of $R$, i.e., is divisible by $\prod_{i=1}^{k} r_{i i}$. 
Thus $\prod_{i=1}^{k} r_{i i}$ is a linear combination of minors and at the same time a divisor of each of those. Hence,

$$
\left|\prod_{i=1}^{k} r_{i i}\right|=\underset{\substack{u \subset\{1, \ldots, m\} \\\|u\|=k}}{\operatorname{GCD}}\left\{\operatorname{det}\left(a_{i j}\right)_{\substack{i \in u \\ j \in\{1, \ldots, k\}}}\right\}=: \tilde{d}
$$

In our case

$$
\begin{array}{r}
\left|\prod_{i=1}^{2} r_{i i}\right|=\operatorname{GCD}\left\{\operatorname{det}\left(\begin{array}{cc}
p_{n} & q_{n} \\
p_{n-1} & q_{n-1}
\end{array}\right), \operatorname{det}\left(\begin{array}{cc}
p_{n} & q_{n} \\
p_{n-2} & q_{n-2}
\end{array}\right), \operatorname{det}\left(\begin{array}{cc}
p_{n-1} & q_{n-1} \\
p_{n-2} & q_{n-2}
\end{array}\right),\right. \\
\left.D_{n} \cdot \operatorname{GCD}\left(p_{n}, p_{n-1}, p_{n-2}, q_{n}, q_{n-1}, q_{n-2}, D_{n}\right)\right\},
\end{array}
$$

which, in view of the assumption $D_{n} \equiv 0 \bmod d_{n}$ (see (1.7)), yields

$$
r_{11} \cdot r_{22}=d_{n}
$$

Part A) of Theorem 1 is proved.

Remark 1. It is clear that the above arguments extend to arbitrary systems of congruences given by the equations

$$
(x_{1}, x_{2}, \ldots, x_{l}, \underbrace{-P,-Q, \ldots,-S}_{k}) A=(\underbrace{0,0, \ldots, 0}_{k}, x_{1}, x_{2}, \ldots, x_{l}),
$$

with matrices

$$
A:=\left(\begin{array}{cccccccc}
\overbrace{p_{n}} & q_{n} & \ldots & s_{n} & \overbrace{1} & 0 & \ldots & 0 \\
p_{n-1} & q_{n-1} & \ldots & s_{n-1} & 0 & 1 & \ldots & 0 \\
\vdots & \vdots & & \vdots & \vdots & \vdots & & \vdots \\
p_{n-l+1} & q_{n-l+1} & \ldots & s_{n-l+1} & 0 & 0 & \ldots & 1 \\
D_{n} & 0 & \ldots & 0 & & & & \\
0 & D_{n} & \ldots & 0 & & & & \\
\vdots & \vdots & & \vdots & & & 0 & \\
0 & 0 & \ldots & D_{n} & & & &
\end{array}\right) .
$$

The determinant of the integral lattice $L$ on vectors $\left(x_{1}, x_{2}, \ldots, x_{l}\right)$ equals (see (3.5))

$$
\operatorname{det}(L)=\frac{D_{n}^{k}}{\tilde{d}}
$$

Proof of B). We begin with the first of the two congruences (1.9). Let $\left\{r_{n}\right\}$ be some linearly independent of $\left\{p_{n}\right\}$ and $\left\{q_{n}\right\}$ solution of the difference equation (1.1). We have

$$
\left\{\begin{array}{l}
p_{n+1}=K_{n}^{(0)} p_{n}+K_{n}^{(1)} p_{n-1}+K_{n}^{(2)} p_{n-2}, \\
q_{n+1}=K_{n}^{(0)} q_{n}+K_{n}^{(1)} q_{n-1}+K_{n}^{(2)} q_{n-2}, \\
r_{n+1}=K_{n}^{(0)} r_{n}+K_{n}^{(1)} r_{n-1}+K_{n}^{(2)} r_{n-2},
\end{array}\right.
$$

where $K_{n}^{(2)}$ is the ratio of consecutive determinants

$$
\left|\begin{array}{ccc}
p_{n} & q_{n} & r_{n} \\
p_{n-1} & q_{n-1} & r_{n-1} \\
p_{n-2} & q_{n-2} & r_{n-2}
\end{array}\right|=: \Delta_{n}, \quad K_{n}^{(2)}=\frac{\Delta_{n+1}}{\Delta_{n}}
$$


Using the explicit coefficients in (1.1) we have, with a suitable normalization of the $\left\{r_{n}\right\}$ (cf. (1.7)):

$$
\Delta_{n}=[(n-1) !]^{2}[(n-2) !]^{2}(16 n-15) .
$$

Expanding $\Delta_{n}$ by the last column, we have the first congruence in (1.9):

$$
\Delta_{n} \equiv 0 \quad \bmod d_{n} .
$$

We now establish the second congruence in (1.9). As was shown in 7], the sequences $\left\{p_{n}\right\}$ and $\left\{q_{n}\right\}$ can be thought of as a result of "thickening" of other sequences whose recurrence relations have integer coefficients and the coefficient of the term with highest index in the relation is always 1 . We shall prove the divisibility properties of the determinant in question using the following representation for the numbers $p_{n}$ and $q_{n}$.

Suppose the sequence of numbers $\tilde{p}_{n}$ and $\tilde{q}_{n}$ is determined by a system of recurrence relations

$$
\begin{aligned}
\tilde{q}_{4 n-1} & =n \tilde{q}_{4 n-3}+\tilde{q}_{4 n-2}, \\
\tilde{q}_{4 n} & =n \tilde{q}_{4 n-4}-n \tilde{q}_{4 n-3}+3 \tilde{q}_{4 n-2}, \quad n=1,2,3, \ldots, \\
\tilde{q}_{4 n+1} & =n \tilde{q}_{4 n-1}+(n+1) \tilde{q}_{4 n}, \\
\tilde{q}_{4 n+2} & =n \tilde{q}_{4 n-2}+(2 n+1)^{2} \tilde{q}_{4 n}-n \tilde{q}_{4 n+1},
\end{aligned}
$$

with initial conditions

$$
\begin{array}{rrr}
\tilde{p}_{0}=0, & \tilde{p}_{1}=1, & \tilde{p}_{2}=1, \\
\tilde{q}_{0}=1, & \tilde{q}_{1}=1, & \tilde{q}_{2}=1 .
\end{array}
$$

Then (see [7), for $p_{n}$ and $q_{n}$ in (1.1) and (1.2) we have $p_{n}=\tilde{p}_{4 n}$ and $q_{n}=\tilde{q}_{4 n}$.

Multiplying the first three relations (3.9) by, respectively, $n, n$ and 1 and adding the results we have, after cancellations,

$$
\tilde{q}_{4 n+1}=\tilde{q}_{4 n}+4 n \tilde{q}_{4 n-2}+n^{2} \tilde{q}_{4 n-4}, \quad n \in \mathbb{N} .
$$

Next we substitute the found relation into the last relation in (3.9) and into the second relation shifted by $n$. We then have relations for the even terms of the sequence with leading coefficients 1 :

$$
\begin{aligned}
& \tilde{q}_{4 n+2}=\left(4 n^{2}+3 n+1\right)^{2} \tilde{q}_{4 n}-n(4 n-1) \tilde{q}_{4 n-2}-n^{3} \tilde{q}_{4 n-4}, \quad n \in \mathbb{N} . \\
& \tilde{q}_{4 n+4}=3 \tilde{q}_{4 n+2}-4 n(n+1) \tilde{q}_{4 n-2}-n^{2}(n+1) \tilde{q}_{4 n-4},
\end{aligned}
$$

We now set

$$
\Delta[a, b](n):=\operatorname{det}\left(\begin{array}{ll}
\tilde{p}_{4 n+a} & \tilde{p}_{4 n+b} \\
\tilde{q}_{4 n+a} & \tilde{q}_{4 n+b}
\end{array}\right) .
$$

Using (3.11) and expanding by the first column we obtain a number of recurrence relations for those determinants:

$$
\begin{aligned}
\Delta[4,2](n) & =4 n(n+1) \Delta[2,-2](n)+n^{2}(n+1) \Delta[2,-4](n), \\
\Delta[4,0](n) & =3 \Delta[2,0](n)+4 n(n+1) \Delta[0,-2](n)+n^{2}(n+1) \Delta[0,-4](n), \\
\Delta[4,-4](n) & =3 \Delta[2,-4](n)-4 n(n+1) \Delta[-2,-4](n), \\
\Delta[2,0](n) & =n(4 n-1) \Delta[0,-2](n)+n^{3} \Delta[0,-4](n), \\
\Delta[2,-2](n) & =\left(4 n^{2}+3 n+1\right) \Delta[0,-2](n)+n^{3} \Delta[-2,-4](n), \\
\Delta[2,-4](n) & =\left(4 n^{2}+3 n+1\right) \Delta[0,-4](n)-n(4 n-1) \Delta[-2,-4](n) .
\end{aligned}
$$


Using substitution, we can express all the quantities in terms of the following three: $\Delta[0,-2](n), \Delta[0,-4](n), \Delta[-2,-4](n)$. Consider the vectors $V_{n}$ with the following coordinates:

$$
V_{n}:=\left(\begin{array}{c}
\Delta[0,-2](n) \\
n \cdot \Delta[0,-4](n) \\
n \cdot \Delta[-2,-4](n)
\end{array}\right) .
$$

Since $\Delta[a, b](n+1)=\Delta[a+4, b+4](n)$, we have

$$
V_{n+1}=n(n+1)\left(\begin{array}{ccc}
4\left(4 n^{2}+3 n+1\right) & 4 n^{2}+3 n+1 & n \\
16 n+1 & 4 n+1 & 0 \\
4 n-1 & n & 0
\end{array}\right) V_{n},
$$

which implies by induction that the components of $V_{n}$ are divisible (we use the notation $\vdots)$ by $n !(n-1) !$. Hence,

$$
\begin{aligned}
\Delta[0,-2](n) \vdots n !(n-1) !, & \Delta[0,-4](n) \vdots[(n-1) !]^{2}, \quad \Delta[-2,-4](n) \vdots[(n-1) !]^{2}, \\
\operatorname{det}\left(\begin{array}{cc}
p_{n-1}, & p_{n-2} \\
q_{n-1}, & q_{n-2}
\end{array}\right) & =\Delta[0,-4](n-1) \vdots[(n-2) !]^{2}, \\
\operatorname{det}\left(\begin{array}{c}
p_{n}, p_{n-2} \\
q_{n}, q_{n-2}
\end{array}\right) & =\Delta[4,-4](n-1) \vdots[(n-2) !]^{2}, \\
\operatorname{det}\left(\begin{array}{c}
p_{n}, p_{n-1} \\
q_{n}, q_{n-1}
\end{array}\right) & =\Delta[0,-4](n) \vdots[(n-1) !]^{2},
\end{aligned}
$$

which establishes the second relation in (1.9).

\section{Estimates on the VeCtors in $L$ AND the Forms $F_{n}$}

In this section we shall prove Proposition 1 and Theorems 2 and 3 (see $₫ 2$ ).

Proof of Proposition 1. We want to find the asymptotics of the determinants in (2.6). To this end, we need the asymptotic formulas (1.3). Notice that the proof of those formulas in [6] implies that for $q_{n}$ and $f_{n}$ there exist asymptotic series in the powers $(1 / \sqrt{n})^{k}$, $k=0,1,2, \ldots$. Therefore

$$
\begin{aligned}
& q_{n}=A \cdot(2 n) ! e^{\sqrt{2 n}} n^{-1 / 4}\left(1+\frac{c_{1}}{\sqrt{n}}+\frac{c_{2}}{n}+o\left(\frac{1}{n}\right)\right), \\
& f_{n}=p_{n}-\gamma q_{n}=B \cdot(2 n) ! e^{-\sqrt{2 n}} n^{-1 / 4}\left(1+\frac{c_{3}}{\sqrt{n}}+\frac{c_{4}}{n}+o\left(\frac{1}{n}\right)\right),
\end{aligned}
$$

where

$$
A:=\frac{1}{\sqrt{\pi}(4 e)^{3 / 8}}, \quad B:=\frac{2 \sqrt{\pi}}{(4 e)^{3 / 8}},
$$

and for the constants $c_{1}, \ldots, c_{4}$ we only need to know that they exist.

Recall the identity

$$
\operatorname{det}\left(\begin{array}{ll}
p_{n-1} & p_{n} \\
q_{n-1} & q_{n}
\end{array}\right)=q_{n}\left(p_{n-1}-\gamma q_{n-1}\right)-q_{n-1}\left(p_{n}-\gamma q_{n}\right) .
$$


Substituting asymptotics (4.1) in it, we have

$$
\begin{aligned}
& \operatorname{det}\left(\begin{array}{cc}
p_{n-1} & p_{n} \\
q_{n-1} & q_{n}
\end{array}\right) \\
&= A B(2 n) !(2 n-2) ! n^{-1 / 4}(n-1)^{-1 / 4} \\
& \times\left[e^{\sqrt{2 n}-\sqrt{2(n-1)}}\left(1+\frac{c_{1}}{\sqrt{n}}+\frac{c_{2}}{n}+o\left(\frac{1}{n}\right)\right)\left(1+\frac{c_{3}}{\sqrt{n-1}}+\frac{c_{4}}{n-1}+o\left(\frac{1}{n}\right)\right)\right. \\
&\left.\quad-e^{\sqrt{2(n-1)}-\sqrt{2 n}}\left(1+\frac{c_{1}}{\sqrt{n-1}}+\frac{c_{2}}{n-1}+o\left(\frac{1}{n}\right)\right)\left(1+\frac{c_{3}}{\sqrt{n}}+\frac{c_{4}}{n}+o\left(\frac{1}{n}\right)\right)\right] \\
&= \frac{A B}{\sqrt{n-\frac{1}{2}}}(2 n) !(2 n-2) !\left(1+\frac{c_{1}}{\sqrt{n}}+\frac{c_{2}}{n}\right)\left(1+\frac{c_{3}}{\sqrt{n}}+\frac{c_{4}}{n}\right) \\
& \times\left(2 \sinh \left(\frac{2}{\sqrt{2 n}+\sqrt{2(n-1)}}\right)+o\left(\frac{1}{n}\right)\right) \\
&= \frac{2 \sqrt{2} A B}{\sqrt{n}}(2 n) !(2 n-2) !\left(1+\frac{c_{1}+c_{3}}{\sqrt{n}}+o\left(\frac{1}{\sqrt{n}}\right)\right) .
\end{aligned}
$$

Similarly,

$$
\begin{aligned}
\operatorname{det}\left(\begin{array}{cc}
p_{n} & p_{n-2} \\
q_{n} & q_{n-2}
\end{array}\right)= & -\frac{A B}{\sqrt{n-1}}(2 n) !(2 n-4) !\left(1+\frac{c_{1}}{\sqrt{n}}+\frac{c_{2}}{n}\right)\left(1+\frac{c_{3}}{\sqrt{n}}+\frac{c_{4}}{n}\right) \\
& \times\left(2 \sinh \left(\frac{4}{\sqrt{2 n}+\sqrt{2(n-2)}}\right)+o\left(\frac{1}{n}\right)\right) \\
= & -\frac{4 \sqrt{2} A B}{n}(2 n) !(2 n-4) !\left(1+\frac{c_{1}+c_{3}}{\sqrt{n}}+o\left(\frac{1}{\sqrt{n}}\right)\right) .
\end{aligned}
$$

Finally,

$$
\operatorname{det}\left(\begin{array}{ll}
p_{n-2} & p_{n-1} \\
q_{n-2} & q_{n-1}
\end{array}\right)=\frac{2 \sqrt{2} A B}{n}(2 n-2) !(2 n-4) !\left(1+\frac{c_{1}+c_{3}}{\sqrt{n}}+o\left(\frac{1}{\sqrt{n}}\right)\right) .
$$

Substituting the obtained formulas in (2.6) we have

$$
\vec{V}_{0}=\frac{A B}{\sqrt{2}} \frac{[(2 n) !]^{2}}{n^{3} d_{n}}\left(\frac{1}{16 n^{4}}+O\left(\frac{1}{n^{9 / 2}}\right), \frac{-1}{2 n^{2}}+O\left(\frac{1}{n^{5 / 2}}\right), 1+O\left(\frac{1}{\sqrt{n}}\right)\right) .
$$

This establishes (2.7). Proposition 1 is proved.

Proof of Theorem 3 , Let $\vec{V}$ be any vector in $L$ not collinear with $\vec{V}_{0}$. For such a vector, either $P$ or $Q$ is nonzero,

$$
\left\langle\vec{V}, \vec{p}^{(n)}\right\rangle=P D_{n}, \quad\left\langle\vec{V}, \vec{q}^{(n)}\right\rangle=Q D_{n} .
$$

Therefore

$$
\|\vec{V}\| \geq \frac{D_{n}}{\max \left(\left\|p_{n}\right\|,\left\|q_{n}\right\|\right)} \sim \frac{D_{n} n^{1 / 4}}{A(2 n) ! e^{\sqrt{2 n}}} .
$$

Now fix (Theorem 3) $D_{n}$ :

$$
D_{n}:=\left[n^{3 / 4} e^{\sqrt{2 n}}\right] k_{n}^{(0)} \Delta_{n} \sim\left[n^{3 / 4} e^{\sqrt{2 n}}\right] \frac{((2 n) !)^{3}}{(n !)^{2}},
$$

and consider a reduced basis of $L$. It is known (see [9]) that the length of a minimal vector $\vec{b}^{(0)}$ in that basis is less than the length of any vector in the lattice multiplied by 
an absolute constant depending on the dimension of the lattice. Using $\vec{V}_{0}$ we have an estimate (see (1.11))

$$
\left\|\vec{b}^{(0)}\right\| \leq 4\left\|\overrightarrow{V_{0}}\right\| \sim 2 \sqrt{2} A B \frac{((2 n) !)^{2}}{n^{3} d_{n}} \lesssim 2 \sqrt{2} A B n \frac{((2 n) !)^{2}}{(n !)^{2}} .
$$

At the same time, substituting (4.3) for $D_{n}$ in the right-hand side of (4.2) we have

$$
\|\vec{V}\| \geq \frac{1}{A} n \frac{((2 n) !)^{2}}{(n !)^{2}} .
$$

As $2 \sqrt{2} A^{2} B<1$, we conclude that $\vec{b}_{0}$ is collinear with $\vec{V}_{0}$ and, since the coordinates of $\vec{V}_{0}$ are relatively prime,

$$
\vec{b}_{0}= \pm \vec{V}_{0}
$$

Since the "minimal" vector and the "annihilating" vector of $L$ coincide, (2.9) turns into

$$
\begin{gathered}
\left\|b^{(1)}\right\| l 2^{3 / 4} \frac{[\operatorname{det}(L)]^{1 / 2}}{\left\|\overrightarrow{V_{0}}\right\|^{1 / 2}} \sim 2^{3 / 4} \frac{D_{n}}{\sqrt{d_{n}}} \frac{2^{1 / 4}}{\sqrt{A B}} n^{3 / 2} \frac{\sqrt{d_{n}}}{(2 n) !}=\frac{2}{\sqrt{A B}} n^{3 / 2} \frac{D_{n}}{(2 n) !}, \\
\left\|b^{(2)}\right\| l 2^{3 / 2} 4 \frac{\operatorname{det}(L)}{\left\|\overrightarrow{V_{0}}\right\|^{2}} \sim 2^{7 / 2} \frac{D_{n}^{2}}{d_{n}} \frac{2}{(A B)^{2}} n^{6} \frac{d_{n}^{2}}{[(2 n) !]^{4}}=\frac{2^{9 / 2}}{(A B)^{2}} n^{6} \frac{D_{n}^{2} d_{n}}{[(2 n) !]^{4}} .
\end{gathered}
$$

Therefore, for forms (2.12) $(j=1,2)$

$$
F_{n}^{(j)}:=P_{n}^{(j)}-\gamma Q_{n}^{(j)}=\frac{1}{D_{n}}\left\langle b^{(j)}, \vec{f}^{(n)}\right\rangle \leq \frac{\left\|b^{(j)}\right\|\left\|\vec{f}^{(n)}\right\|}{D_{n}},
$$

we have, in view of (1.3), the following estimates:

$$
\begin{aligned}
& \left|F_{n}^{(1)}\right| l \frac{2}{\sqrt{A B}} n^{3 / 2} \frac{D_{n}}{(2 n) !} \frac{\left\|\vec{f}^{(n)}\right\|}{D_{n}}=\frac{2}{\sqrt{A B}} \frac{n^{3 / 2}}{(2 n) !}\left\|\vec{f}^{(n)}\right\| \sim 2 \sqrt{\frac{B}{A}} n^{5 / 4} e^{-\sqrt{2 n}}, \\
& \left|F_{n}^{(2)}\right| l \frac{2^{5 / 2}}{(A B)^{2}} n^{6} \frac{D_{n} d_{n}}{[(2 n) !]^{4}}\left\|\vec{f}^{(n)}\right\| \lesssim \frac{3 \cdot 2^{5 / 2}}{A^{2} B} n^{10.5} \frac{[(2 n) !]^{3}(n !)^{2}(2 n) !}{(n !)^{2}[(2 n) !]^{4}} \sim O\left(n^{10.5}\right),
\end{aligned}
$$

which proves Theorem 3 .

Proof of Theorem 2, We now fix (Theorem 2) $D_{n}$ :

$$
D_{n}:=k_{n}^{(0)} \Delta_{n} \sim \frac{[(2 n) !]^{3}}{(n !)^{2}}
$$

and assume (4.4) holds (which does not contradict (2.8)). Again, we can estimate two other vectors of the reduced basis as in (4.5). The estimate for $\left|F_{n}^{(1)}\right|$ (under the assumptions of Theorem 1) does not depend on $D_{n}$. For $\left|F_{n}^{(2)}\right|$, taking into account the new $D_{n}$, we have

$$
\left|F_{n}^{(2)}\right| \leq \frac{2^{5 / 2}}{(A B)^{2}} n^{6} \frac{D_{n} d_{n}}{[(2 n) !]^{4}}\left\|\vec{f}^{(n)}\right\| \lesssim \frac{3 \cdot 2^{5 / 2}}{A^{2} B} n^{9.75} \frac{[(2 n) !]^{3}(n !)^{2}(2 n) !}{(n !)^{2}[(2 n) !]^{4}} e^{-\sqrt{2 n}} .
$$

Theorem 2 is proved. 


\section{REFERENCES}

[1] A. I. Aptekarev (ed.), Rational approximation of Euler's constant and recurrence relations, Current Problems in Math., vol. 9, Steklov Math. Inst. RAN, 2007. (Russian)

[2] A. I. Aptekarev, A. Branquinho, and W. Van Assche, Multiple orthogonal polynomials for classical weights, Trans. Amer. Math. Soc. 355 (2003), no. 10, 3887-3914. MR.1990569 (2004g:33014)

[3] D. V. Khristoforov, Recurrence relations for the Hermite-Padé approximants of a system of four functions of Markov and Stieltjes type, in [1, pp. 11-26. (Russian)

[4] A. I. Bogolyubskii, Recurrence relations with rational coefficients for some jointly orthogonal polynomials defined by Rodrigues' formula, in [1, pp. 27-35. (Russian)

[5] A. I. Aptekarev and D. N. Tulyakov, Four-term recurrence relations for $\gamma$-forms, in 1]. pp. 37-43. (Russian)

[6] A. I. Aptekarev and V. G. Lysov, Asymptotics of $\gamma$-forms jointly generated by orthogonal polynomials, in [1]. pp. 55-62. (Russian)

[7] D. N. Tulyakov, A system of recurrence relations for rational approximants of the Euler constant, Mat. Zametki (to appear).

[8] T. Rivoal, Rational approximations for values of derivatives of the gamma function, http://wwwfourier.ujf-grenoble.fr/rivoal/articles.html.

[9] A. K. Lenstra, H. W. Lenstra, and L. Lovasz, Factoring polynomials with rational coefficients, Math. Ann. 261 (1982), no. 4, 515-534. MR682664 (84a:12002)

M. V. Keldysh Institute of Applied Mathematics, 4 Miusskaya Ploshchad', Moscow 125047, Russia, and Lomonosov State University, Moscow, Russia

E-mail address: aptekaa@keldysh.ru

M. V. Keldysh Institute of Applied Mathematics, 4 Miusskaya Ploshchad', Moscow 125047 , Russia

Translated by ALEX MARTSINKOVSKY 